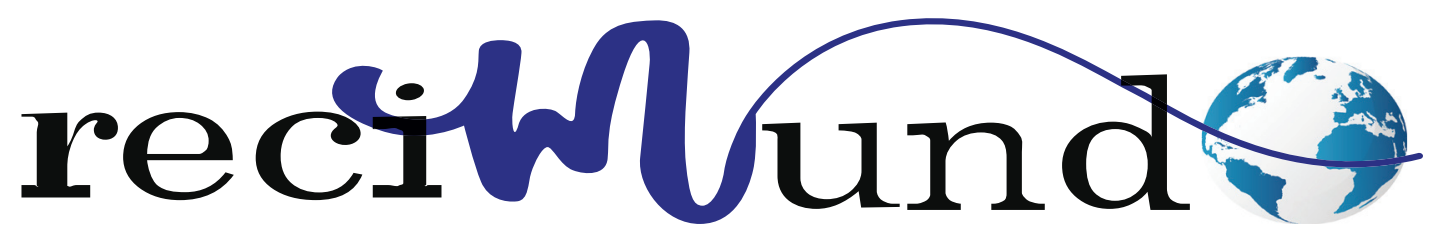

Revista Científica Mundo de la Investigación y el Conocimiento

DOI: $10.26820 /$ recimundo/5.(4).dic.2021.332-343

URL: https://recimundo.com/index.php/es/article/view/1373

EDITORIAL: Saberes del Conocimiento

REVISTA: RECIMUNDO

ISSN: 2588-073X

TIPO DE INVESTIGACIÓN: Artículo de investigación

CÓdIGO UNESCO: 32 Ciencias Médicas

PAGINAS: $332-343$

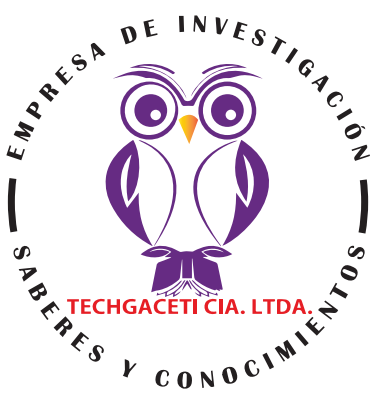

\title{
Valores de la temperatura en pacientes pediátricos y adultos mayores. "un enfoque de revisión"
}

Temperature values in pediatric and elderly patients. "A review approach" Valores de temperatura em pacientes pediátricos e idosos. "Uma abordagem de revisão"

Sandra Maritza Mena Sánchez¹, Verónica Sofía Quenorán Almeida²

RECIBIDO: 25/09/2021 ACEPTADO: 10/10/2021 PUBLICADO: 30/10/2021

1. Estudiante de décimo semestre de Enfermería; Universidad Técnica de Ambato; Ecuador; smena3048@uta. edu.ec; (iD https://orcid.org/0000-0002-4187-2089

2. Licenciada en Enfermería; Magister en Gerencia de Salud para el Desarrollo Local; Docente de la Universidad Técnica de Ambato; Facultad Ciencias de la Salud; Ecuador; iD https://orcid.org/0000-0002-1870-0887

CORRESPONDENCIA

Sandra Maritza Mena Sánchez

smena3048@uta.edu.ec

Ambato; Ecuador

๑ 


\section{RESUMEN}

El objetivo de este artículo de revisión es comparar los valores de la temperatura corporal en pacientes pediátricos y adultos mayores y los factores que influyen en su valor durante la medición. Corresponde a una investigación cuantitativa con diseño basado en la revisión sistemática de literatura y metaanálisis. Se analizaron en total 34 artículos encontrados en bases de datos especializadas como Springer, Scielo, Dialnet, Elsevier, Latindex y publicados entre el año 2017 al 2021. Para la demostración de los principales hallazgos se optimizaron tablas de análisis y listas de cotejo, integrando así los resultados de varios estudios independientes homólogos al tema central. Finalmente se pudo concluir que la temperatura es un signo vital variable que oscila entre 36 y $38^{\circ} \mathrm{C}$, y que depende de factores como: la edad de la persona, el sitio de medición, el género, el, y también el tipo de dispositivo a usar.

Palabras clave: Adultos mayores, Factores, Pacientes pediátricos, Temperatura corporal Valores normales.

\section{ABSTRACT}

The aim of this review article is to compare body temperature values in pediatric and elderly patients. In addition, the factors influencing the value during the measurement will be identified. It corresponds to a qualitative research with a design based on a systematic literature review and meta-analysis. 34 articles found in specialized databases such as Springer, Scielo, Dialnet, Elsevier, Latindex and published between 2017 and 2021 were analyzed. To demonstrate the main findings, analysis tables and checklists were optimized, thus integrating the results of several independent studies homologous to the central theme. Finally, he concluded that temperature is a variable vital sign that ranges between 35 and 38 degrees Celsius and depends on factors such as the age of the person, the site of measurement, gender, time of day and the device that will be used.

Keywords: Elderly people, Factors, Pediatric patients, Body temperature Normal values.

\section{RESUMO}

O objetivo deste artigo de revisão é comparar os valores da temperatura corporal em pacientes pediátricos e idosos. Além disso, os fatores que influenciam o valor durante a medição serão identificados. Corresponde a uma pesquisa qualitativa com desenho baseado em revisão sistemática da literatura e meta-análise. Foram analisados 34 artigos encontrados em bases de dados especializadas como Springer, Scielo, Dialnet, Elsevier, Latindex e publicados entre 2017 e 2021. Para demonstrar os principais achados, foram otimizadas tabelas de análise e checklists, integrando os resultados de diversos estudos independentes homólogos ao tema central. Por fim, concluiu que a temperatura é um sinal vital variável que varia entre 35 e 38 graus Celsius e depende de fatores como a idade da pessoa, o local da medição, o sexo, a hora do dia e o aparelho que será utilizado.

Palavras-chave: Idosos, Fatores, Pacientes pediátricos, Temperatura corporal Valores normais. 


\section{Introducción}

Los signos vitales se definen como una medida objetiva de las funciones fisiológicas para la evaluación clínica ${ }^{1}$. El estado de salud de un individuo se refleja en estos indicadores de la función corporal, se considera que la medición de la temperatura, presión arterial, oximetría de pulso, frecuencia cardíaca y respiratoria deben ser procedimientos verificables, y no automáticos ni rutinarios; aplicando las diferentes técnicas con pleno conocimiento de sus factores que los pudieran modificar variando con ellos sus rangos o valores ${ }^{2,3}$.

Se entiende por temperatura corporal a la diferencia entre la actividad de calor producida por el cuerpo y la que se libera al ambiente por la misma medida en grados. Los procesos metabólicos de los tejidos centrales del cuerpo son los que generan el calor trasferido a la superficie de la piel por medio de la sangre circulante y luego se disipa al ambiente ${ }^{4}$. La temperatura corporal central (intracraneal, intratorácica e intraabdominal) es más alta que de la superficie del cuerpo, la temperatura corporal normal es de $36.5^{\circ} \mathrm{C}$ y los $37.2^{\circ} \mathrm{C}$, mientras que para las personas de la tercera edad se encuentra entre los $36.0^{\circ} \mathrm{C}$ a $37.0^{\circ} \mathrm{C}$ y en pacientes pediátricos su temperatura va de $36.5^{\circ} \mathrm{C}$ a $37.5^{\circ} \mathrm{C}$. Las lecturas de la temperatura rectal son 0,5 grado más altas que las orales ${ }^{5,1}$.

En la actualidad los mejores instrumentos para el control de temperatura son los termómetros digitales para uso axilar, oral o rectal $^{6}$, a pesar de que han ido cobrando más jerarquía los termómetros infrarrojos óticos, así como los infrarrojos cutáneos, los cuales permiten lograr mediciones precisas, válidas y confiables, útiles para manifestar cualquier variación y poder tomar medidas oportunas ${ }^{7}$.

Hay variaciones normales de temperatura en dependencia de la edad, género, actividad física, estado de salud e incluso temperaturas ambientales. La temperatura corporal se valora y compara con rangos normales aceptados y con los patrones habituales del paciente en una amplia variedad de circunstancias ${ }^{8}$. La atención cuidadosa a los detalles en la toma de este signo y la exactitud en la interpretación de sus valores son de extrema importancia, ya que al presentarse un cambio podría indicar una modificación en el estado de salud de la persona ${ }^{9}$.

La medición de la temperatura corporal permite entonces, la pronta detección de diferentes complicaciones clínicas, que de manifestarse pueden afectar al sistema cardíaco, respiratorio, tegumentario, digestivo, inmunológico, originando una hipertermia maligna ${ }^{10}$, donde las consecuencias serían una posible hipotensión, escalofríos, confusión, delirio y convulsiones sobre todo en la infancia ${ }^{11}$.

En cambio, en los recién nacidos la hipotermia es preocupante, ya que es bien conocido que las implicaciones clínicas pueden generar un daño cerebral y neurológico importante en el niño. Se determina que un rango de hipotermia se encuentra entre $32.0^{\circ} \mathrm{C}$ a $35.9^{\circ} \mathrm{C}$, requiriendo de manera urgente el calentamiento del paciente ${ }^{12}$. Además, como ya se mencionó la temperatura corporal superior a los $38^{\circ} \mathrm{C}$ en recién nacido, provoca convulsiones por picos febriles $^{13}$.

Por otro lado, los episodios febriles son más frecuentes en pacientes pediátricos donde la hipertermia es superior a los $41^{\circ} \mathrm{C}$, de igual manera, son más propensos a sufrir crisis epiléptica debido a la fiebre alta no controlada ${ }^{1}$. La sintomatología común que produce la hipertermia es pérdida de conciencia, rigidez corporal, taquicardia y bradipnea ${ }^{14}$.

Así que, los adultos mayores son más vulnerables y susceptibles a presentar cambios en la temperatura corporal, ya que esta es 
más baja en comparación con los adultos más jóvenes, debido a una alteración en el sistema regulador, esto se debe a la disminución de masa y tonicidad muscular, también al cambio de ambiente, vivienda y vestimenta ${ }^{15}$. Es frecuente que el adulto mayor desarrolle alguna enfermedad que eleve la temperatura pero que no desencadena fiebre, complicando la condición clínica del anciano, esto debido a que su temperatura no alcanza lo que tradicionalmente se conoce como hipertermia, por este motivo se debe usar rangos de temperatura más bajos de $37,1^{\circ} \mathrm{C}$ como referencia ${ }^{16}$.

En el contexto del proceso enfermero en la fase de valoración se deben determinar los factores relacionados para emitir el diagnóstico de enfermería basado en el estado actual del paciente, cuyos referentes pueden incluir hipertermia, hipotermia, termorregulación ineficaz y/o riesgo de temperatura corporal desequilibrada ${ }^{17}$. Los resultados que se espera alcanzar cuando se realiza la valoración de la temperatura es que se aplique la técnica con exactitud sin causar ninguna lesión y que el paciente experimente mínimas molestias ${ }^{18}$.

Sin duda alguna, en términos administrativos, la temperatura es un signo confiable y eficaz, ya que su costo es económicamente bajo con respecto a otros signos de monitoreo que requieren equipos biomédicos de alto valor, costes por mantenimiento y su tiempo de vida útil es relativamente corto $^{19}$.

Por consiguiente, la presente investigación tiene como objetivo comparar los valores de la temperatura corporal en pacientes pediátricos y adultos mayores, presentando datos comparativos de los rangos normales según su autor y además identificar factores que influyen en la medición y alteran el resultado de la temperatura.

La investigación tiene como propósito brindar valores de la temperatura corporal normal y anormal según la información recopi- lada de diversos autores, además ayudará a identificar factores importantes que alteran sus resultados y que pueden no considerarse por su facilitada rutina para su valoración.

\section{Metodología}

El presente artículo corresponde a una investigación cuantitativa con diseño basado en la revisión sistemática de literatura y metaanálisis. Con ello, se definió una población de 633 artículos con palabras clave asociadas al tema base, pero finalmente se seleccionaron 34 para su análisis ya que se consideraron relevantes para el cumplimiento del objetivo. Los artículos corresponden a revistas que se encuentran en bases de datos especializadas como Springer, Scielo, Dialnet, Elsevier, Latindex. Como criterios de exclusión se puede señalar que no fueron tomados en cuenta aquellos trabajos investigativos que se encuentran publicados fuera del rango definido entre 2017 al 2021. Según su alcance se considera descriptiva ya que la información analizada se estructuró en dos subapartados: uno dedicado a pacientes pediátricos y otro a adultos mayores.

Para la demostración de los principales hallazgos se optimizaron tablas de análisis y listas de cotejo, integrando así los resultados de varios estudios independientes homólogos al tema central. Las tablas obtenidas se denominan: Tabla $N^{\circ} 1$. Valores normales y anormales de la temperatura en los pacientes pediátricos y adultos mayores; Tabla N²: principales complicaciones con la variable temperatura; Tabla No3: Valores normales según el sitio de medición; Tabla N4: La eficiencia, eficacia y efectividad del dispositivo en la toma de temperatura corporal; encontrándose varianza significativa de los rangos de temperatura corporal con relación con la edad, ciclo de vida, tipo de dispositivo, técnica y entorno. 


\section{Resultados y discusión}

Luego del análisis de la información encontrada en la revisión bibliográfica, se han obtenido los siguientes resultados:

Tabla 1. Valores normales y anormales de la temperatura en los pacientes pediátricos y adultos mayores.

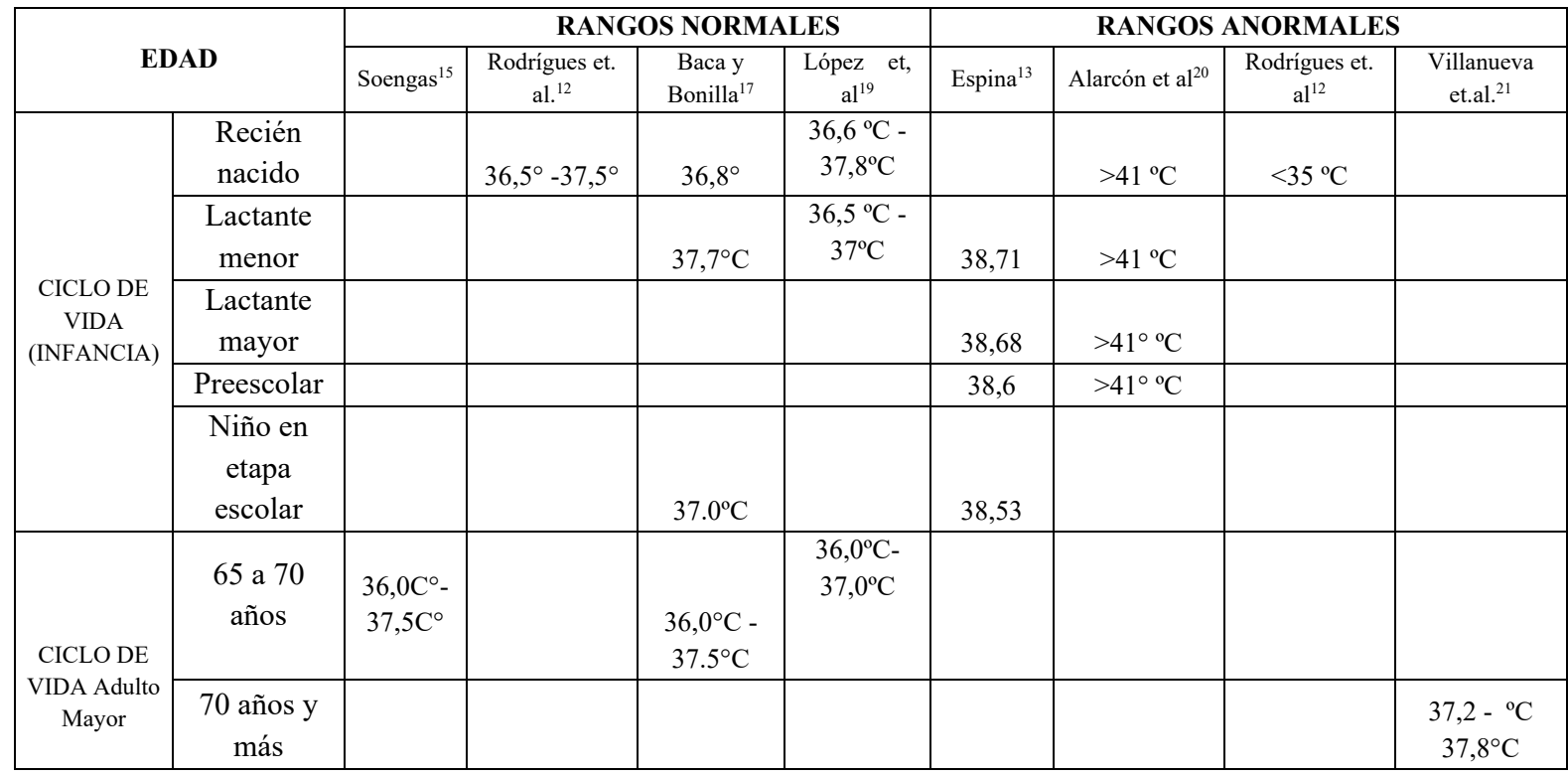

Fuente: Elaboración propia $15,17,1,13,20,21,12$

Con respecto, a la tabla $\mathrm{N}^{\circ} 1$, se cotejo los valores normales y anormales de la temperatura corporal en los pediátricos y adultos mayores, según diferentes autores. En primer lugar, como se demuestra claramente se comparó con la edad en función al ciclo de vida; Donde, López ${ }^{1}$ parte de una temperatura normal baja en los recién nacidos de $36,6^{\circ} \mathrm{C}$ y una temperatura normal alta de $37,8^{\circ} \mathrm{C}$, a diferencia de Rodrigues ${ }^{12}$, indica que una temperatura normal baja es de $36,5^{\circ} \mathrm{C}$ y una temperatura normal alta es de $37,5^{\circ} \mathrm{C}$.

Investigadores como Baca y Bonilla ${ }^{17}$ inciden que una temperatura corporal normal en el lactante menor es de $37,7^{\circ} \mathrm{C}$, para ${ }^{1}$ es de 36,5 y $37,0^{\circ} \mathrm{C}$. Sin embargo, Romero ${ }^{13}$ plantea que una temperatura de $38,71^{\circ} \mathrm{C}$ en el lactante menor y una temperatura de $38,68{ }^{\circ} \mathrm{C}$ en el lactante mayor son rangos anormales, al igual que, una temperatura de $38,6^{\circ} \mathrm{C}$ en preescolares y $38,53^{\circ} \mathrm{C}$ en niños en etapa escolar ${ }^{13}$, igualmente cuando es superior a los $41^{\circ} \mathrm{C}^{20}$.
Por otra parte, en el adulto mayor de 65 a 70 años afirma Soengas ${ }^{15}$ que una temperatura normal baja es de $36,0^{\circ} \mathrm{C}$ y una temperatura normal alta es de $37.5^{\circ} \mathrm{C}$. De igual manera, López ${ }^{1}$ destaca que los valores normales oscilan entre $36.0^{\circ} \mathrm{C}$ a $37.0^{\circ} \mathrm{C}$. En cambio en el adulto mayor de 70 años según Villanue$v^{21}{ }^{21}$, están a partir de los $37,2^{\circ} \mathrm{C}$ y $37,8^{\circ} \mathrm{C}$.

La temperatura del cuerpo humano es un signo vital muy variable y está influenciado por diversos factores, principalmente la edad de la persona ${ }^{4}$. La revisión basada en evidencias proporciona rangos de temperatura normales en pediátricos, una elevación por encima de los $38^{\circ} \mathrm{C}$ en niños es frecuente y se debe muchas de las veces a infecciones ${ }^{13}$. Por el contrario en los adultos mayores el descenso es común debido a la disminución de la capacidad para regular la temperatura corporal, por ese motivo se debe utilizar rangos más bajos de $37,5^{\circ} \mathrm{C}^{15}$. 
Tabla 2. Principales complicaciones con la variable temperatura.

\begin{tabular}{|c|c|c|c|c|c|c|}
\hline Autores & $\begin{array}{c}\text { Banda et } \\
\text { al. }^{32}\end{array}$ & $\begin{array}{c}\text { Rodrigues } \\
\text { et. al. }{ }^{12}\end{array}$ & Storch $^{22}$ & Soengas ${ }^{15}$ & $\begin{array}{c}\text { Romero et } \\
\text { al. }^{14}\end{array}$ & Picón et. $\mathrm{al}^{10}$ \\
\hline Complicaciones & $\begin{array}{l}\text { Recién } \\
\text { nacido }\end{array}$ & $\begin{array}{l}\text { Recién } \\
\text { Nacido }\end{array}$ & Pediátricos & $\begin{array}{l}\text { Adultos } \\
\text { mayores }\end{array}$ & $\begin{array}{l}\text { Adultos } \\
\text { mayores }\end{array}$ & $\begin{array}{l}\text { Adultos } \\
\text { mayores }\end{array}$ \\
\hline Hipotermia severa & & $<32,0^{\circ}$ & $<28^{\circ} \mathrm{C}$. & & & \\
\hline $\begin{array}{c}\text { Hipotermia } \\
\text { moderada }\end{array}$ & & $\begin{array}{l}32,0^{\circ}- \\
35,9^{\circ} \mathrm{C}\end{array}$ & $\begin{array}{c}28^{\circ} \mathrm{C} \text { y } \\
32^{\circ} \mathrm{C} .\end{array}$ & & $\begin{array}{l}32,0^{\circ} \mathrm{C}- \\
33,9^{\circ} \mathrm{C}\end{array}$ & \\
\hline Hipotermia leve & & $\begin{array}{l}36,0^{\circ}- \\
36,4^{\circ} \mathrm{C}\end{array}$ & $\begin{array}{l}32^{\circ} \mathrm{C} \mathrm{y} \\
35^{\circ} \mathrm{C} .\end{array}$ & $<35^{\circ} \mathrm{C}$ & $\begin{array}{l}34,0^{\circ} \mathrm{C}- \\
35,9^{\circ} \mathrm{C}\end{array}$ & $\begin{array}{c}35,0^{\circ} \mathrm{C}-35,5 \\
{ }^{\circ} \mathrm{C}\end{array}$ \\
\hline Hipertermia leve & & & & $>37,5^{\circ} \mathrm{C}$ & & \\
\hline Hipertermia & $>37,5^{\circ} \mathrm{C}$ & & & $>38^{\circ} \mathrm{C}$ & $>37,5^{\circ} \mathrm{C}$ & $>38,3^{\circ} \mathrm{C}$ \\
\hline $\begin{array}{c}\text { Hipertermia } \\
\text { maligna }\end{array}$ & & & & $>40^{\circ} \mathrm{C}$ & & \\
\hline
\end{tabular}

Fuente: Elaboración propia ${ }^{13,12,22,15,1,10}$

Con concordancia, a la tabla №2 sobre las complicaciones, los diferentes autores lo dividen según la edad, por ejemplo, Rodrigues $^{12}$ consideran que se trata de una hipotermia leve, cuando los valores de la temperatura son de $36,0^{\circ} \mathrm{C}$ y $36,4^{\circ} \mathrm{C}$ en los recién nacidos. Una hipotermia moderada dentro de $32^{\circ} \mathrm{C}$ a $35,9^{\circ} \mathrm{C}$, y por debajo del $32^{\circ} \mathrm{C}$ señala como hipotermia severa. Por el contrario, una hipertermia se sitúa por encima de los $37,5^{\circ} \mathrm{C}^{13}$.

Storch $^{22}$ expone que, los rangos de la hipotermia leve en pediátricos son de $32^{\circ} \mathrm{C}$ y $35^{\circ} \mathrm{C}$. Una hipotermia modera es de $28^{\circ} \mathrm{C}$ a $32^{\circ} \mathrm{C}$. Mientras tanto, la hipotermia severa se encuentra por debajo de los $28^{\circ} \mathrm{C}$.

De acuerdo con, Romero ${ }^{14}$ en los adultos mayores se trata de una hipotermia leve, cuando los valores de la temperatura son de $34,0^{\circ} \mathrm{C}$ a $35,9^{\circ} \mathrm{C}$, y una hipotermia moderada es de $32,0^{\circ} \mathrm{C}$ a $33,9^{\circ} \mathrm{C}$. Por otra parte, una temperatura mayor de $37,5^{\circ} \mathrm{C}$ lo denomina como hipertermia; (Picón ${ }^{10}$, Soengas $^{15}$ ) establecen que es superior a los 38 ${ }^{\circ} \mathrm{C}$. Además, la hipertermia maligna sobrepasa los $40^{\circ} \mathrm{C}$.

Una alteración de la temperatura corporal, siendo un descenso (hipotermia) o elevación (hipertermia) puede afectar al sistema cardíaco, respiratorio, tegumentario, digestivo, inmunológico y al no ser identificado o tratado pone en riesgo la vida de la persona. En pediátricos y en adultos mayores se considera hipotermia cuanto el valor es menor $36^{\circ} \mathrm{C}$, e hipertermia cuando sobrepasa $\operatorname{los} 37,5^{\circ} \mathrm{C}^{11,15,10}$.

Tabla 3. Valores normales de la temperatura según el sitio de medición.

\begin{tabular}{|l|c|c|c|}
\hline \multicolumn{4}{|c|}{ Valores normales } \\
\hline \multicolumn{1}{|c|}{ Autor } & $\begin{array}{c}\text { Reyes et al. } \\
\text { Sánchez }\end{array}$ & Quispe & \multicolumn{1}{c|}{$\begin{array}{c}\text { Arias }^{24}, \\
\text { Villanueva }^{26}\end{array}$} \\
\cline { 2 - 4 } & Recién nacido & Pediátricos & 65 años \\
\hline Indicador & $36,0-36,5^{\circ} \mathrm{C}$ & & $36.7^{\circ} \mathrm{C}-37.2^{\circ} \mathrm{C}$ \\
\hline Oral & $36,5^{\circ} \mathrm{C} 37,5^{\circ} \mathrm{C}$ & & $36.2^{\circ} \mathrm{C}-36.7^{\circ} \mathrm{C}$ \\
\hline Axilar & $37,5^{\circ} \mathrm{C}$ & $37,1^{\circ} \mathrm{C}$ & $37.2^{\circ} \mathrm{C}-37.7^{\circ} \mathrm{C}$ \\
\hline Rectal & & $37,0^{\circ} \mathrm{C}$ & $37,2^{\circ} \mathrm{C}-37,8^{\circ} \mathrm{C}$ \\
\hline Timpánica & & &
\end{tabular}

Fuente: Elaboración propia $23,24,25,21$ 
La temperatura corporal está influenciada por el sitio de medición, se investigó las temperaturas promedio en orden de edad y los rangos normales de mediciones orales, rectales, axilares y timpánicas, como se observa en la tabla No3 Reyes ${ }^{23}$, \& Sánchez ${ }^{18}$ concuerdan que en los recién nacidos el valor en la toma oral es de $36,0^{\circ} \mathrm{C} \mathrm{a}$ $36,5^{\circ} \mathrm{C}$, axilar de $36.5^{\circ} \mathrm{C}$ a $37.5^{\circ} \mathrm{C}$ y rectal de $37,5^{\circ} \mathrm{C}$ está sigue siendo la más recomendada por su exactitud, pero se la tomar axilar. En cuanto para, Quispe ${ }^{24}$, la temperatura rectal en pediátricos es de $37,1^{\circ} \mathrm{C}$ y la timpánica de $37,0^{\circ} \mathrm{C}$. La medición rectal sigue siendo la indicada para esa edad pero de igual manera, se puede medir axilar o timpánica ${ }^{24}$.

En los adultos mayores Arias $^{25}$ Storch $^{21}$,enfatizan que la temperatura normal oral está entre los $36.7^{\circ} \mathrm{C}$ y $37.2^{\circ} \mathrm{C}$, axilar de $36.2^{\circ} \mathrm{C}$ a $36.7^{\circ} \mathrm{C}$, rectal de $37.2^{\circ} \mathrm{C}$ a $37.7^{\circ} \mathrm{C}$ y la timpánica de $37,2^{\circ} \mathrm{C}$ a $37,8^{\circ} \mathrm{C}$. A su vez, la medición en el tímpano es seguro, fiable por su cercanía a la carótida y el hipotálamo.

La zona de medida de la temperatura (oral, rectal, timpánica, arteria temporal, esofágica, arterial pulmonar, axilar o incluso vesical urinaria) es un factor que determina la temperatura del paciente. Las medidas invasoras, como la obtenida con un catéter en una arteria pulmonar, se consideran temperaturas centrales; mientras que las temperaturas axilares son reflejo de la temperatura superficial del cuerpo. Es importante recordar que una medida fiable de la temperatura corporal en un solo lugar permite vigilar los patrones de temperatura corporal en el paciente.

Tabla 4. Factores ambientales que influyen en la temperatura corporal.

\begin{tabular}{|c|c|c|c|}
\hline \multicolumn{2}{|c|}{ Autor } & $\operatorname{Duran}^{2}$ & $\operatorname{Dan}^{28}$ \\
\hline \multirow{2}{*}{$\begin{array}{c}\text { Adultos } \\
\text { Mayores }\end{array}$} & Mañana & $36,0{ }^{\circ} \mathrm{C}$ & $36.8{ }^{\circ} \mathrm{C}$ \\
\cline { 2 - 4 } & Tarde & $37,2^{\circ} \mathrm{C}$ & $37.2{ }^{\circ} \mathrm{C}$ \\
\hline \multicolumn{2}{|c|}{ Autor } & Thiel $(27)$ & \\
\hline \multirow{3}{*}{ Pediátricos } & Mañana & $36,6^{\circ} \mathrm{C}$ & \\
\hline \multicolumn{2}{|c|}{ Autor } & $37.6{ }^{\circ} \mathrm{C}$ & \\
\hline \multirow{3}{*}{ Recién nacido } & Tarde & Marzuillo (26) & \\
\cline { 2 - 4 } & Tarde & $36,1^{\circ} \mathrm{C}$ & \\
\hline
\end{tabular}

Fuente: Elaboración propia'2,26,27,28

En cuanto a las variaciones de la temperatura en relación con el tiempo de medición y la edad. Se muestra en la tabla $N^{\circ} 4$, se observa una varianza a través de los valores durante el día ${ }^{26}$. La temperatura normal por la mañana en un recién nacido es de $36,1^{\circ} \mathrm{C}$ y por la tarde de $37,8^{\circ} \mathrm{C}$. En el pediátrico según, Quispe ${ }^{24}$ la temperatura es de $36,6^{\circ} \mathrm{C}$ en la mañana y de $37,6^{\circ} \mathrm{C}$ en la tarde.

Por otro lado, Duran² expone que en los adultos mayores la temperatura por la mañana es de $36,0^{\circ} \mathrm{C}$ y por la tarde es de 37,2
${ }^{\circ} \mathrm{C}$. Conforme a Dan ${ }^{28}$ comienza con una temperatura de $36,8^{\circ} \mathrm{C}$ y en la tarde es de $37,2^{\circ} \mathrm{C}$. En efecto se saca un rango de referencia entre estas dos temperaturas dando un promedio que podría ser por la mañana de $36,4{ }^{\circ} \mathrm{C}$ y por la tarde de $37,2^{\circ} \mathrm{C}$.

El momento del día también influye en la temperatura corporal, de modo que la menor temperatura tiene lugar a las por la mañana 4 y 5 a.m. y más altas al finalizar la tarde 4 y 8 p.m $m^{27,2}$. 
A pesar de extremos en las condiciones ambientales y la actividad física, los mecanismos de control de la temperatura de los seres humanos mantienen la temperatura central (temperatura de los tejidos profundos) relativamente constante. Sin embargo, la temperatura superficial varía, dependien- do del flujo sanguíneo en la piel y de la cantidad de calor perdida hacia el ambiente exterior. Los tejidos y las células corporales actúan de forma eficiente dentro de un intervalo estrecho, entre 36 y $38^{\circ} \mathrm{C}$, pero ninguna temperatura es normal en todas las personas.

Tabla 5. Eficiencia-eficacia-efectividad del dispositivo en la toma de temperatura.

\begin{tabular}{|c|c|c|c|c|}
\hline Autores & Quispe, $\mathrm{H}^{24}$ & $\begin{array}{c}\text { Fernández et } \\
\text { al. }^{29}\end{array}$ & Zarate $^{31}$ & Alatrista, M. et al. \\
\hline \multicolumn{5}{|c|}{ TERMÓMETROS } \\
\hline Mercurio & \begin{tabular}{ll} 
Causan & \multicolumn{2}{c}{ efectos } \\
tóxicos & sobre la \\
persona y & el \\
ambiente debido \\
al mercurio
\end{tabular} & $\begin{array}{lr}\text { No } & \text { son } \\
\text { utilizados } & \text { ya } \\
\text { que es nocivo } \\
\text { para el medio } \\
\text { ambiente y la } \\
\text { salud. }\end{array}$ & & $\begin{array}{l}\text { Se mide en minutos, pero es } \\
\text { peligroso para la salud de } \\
\text { los trabajadores y los } \\
\text { pacientes. }\end{array}$ \\
\hline Digitales & $\begin{array}{lr}\text { Efectivo } & \text { en } \\
\text { cualquier } & \text { rango } \\
\text { de edad } & \end{array}$ & & & \\
\hline $\begin{array}{l}\text { Infrarrojos } \\
\text { de oído }\end{array}$ & $\begin{array}{l}\text { Rápido, edades de } \\
2 \text { a } 6 \text { años }\end{array}$ & & $\begin{array}{l}\text { No son } \\
\text { confiables } \\
\text { en menores de } \\
6 \text { meses, }\end{array}$ & Se mide en segundos \\
\hline
\end{tabular}

Fuente: Elaboración propia 24,29,31,30

En la tabla $\mathrm{N}^{0} 5$ se encuentran investigaciones acerca de la eficiencia-eficacia y efectividad de los diferentes dispositivos con los que se toma la temperatura, los siguientes autores (Fernández ${ }^{29}$, Quispe ${ }^{24}$, mencionan que, los termómetros de mercurio causan efectos tóxicos a pacientes, personal de salud y al ambiente, por ese motivo en la actualidad está prohibido su uso, a pesar de que las tomas son exactas a diferencia de los demás dispositivos. Según Quispe ${ }^{24}$, considera que los termómetros de oído son rápidos y se pueden utilizar en niños de 2 a 6 años. En cambio, los digitales sirven en cualquier rango de edad.
En la medición de la temperatura se usa diversos equipos para obtener una medición exacta también, hay que elegir el sitio más adecuado y el termómetro apropiado basado en la condición del paciente y según la edad. Los de mercurios son fiables puede tardar hasta 5 minutos en dar el resultado, pero están prohibidos debido a su toxicidad; los infrarrojos de oídos se utilizan solo a partir de los dos años. Los termómetros digitales son los más usados ya que sirven para cualquier rango de edad, además son rápidos y fiables $30,29.24$. 
Tabla 6. Género y valores de la temperatura corporal.

\begin{tabular}{|c|c|c|}
\hline \multicolumn{2}{|c|}{ Autor } & $\begin{array}{c}\text { Rivadeneyra, } \\
\text { et al. } .^{34}\end{array}$ \\
\hline \multirow{4}{*}{ Género } & Masculino & $36,5-37^{\circ} \mathrm{C}$ \\
\cline { 2 - 3 } & Femenino & $37,5^{\circ} \mathrm{C}$ \\
\hline
\end{tabular}

Fuente: Elaboración propia ${ }^{34}$

En lo referente a la temperatura según el género, de forma complementaria y no menos relevante, en la tabla $\mathrm{N}^{\circ} 6$ se muestran que las mujeres tienen un incremento en la elevación de la temperatura corporal, esto se debe al cambio del ciclo menstrual de modo que es $0,5^{\circ} \mathrm{C}$ más alta en la fase lútea ${ }^{34}$.

No existe una sola temperatura corporal que pueda considerarse normal, por ejemplo, en una mujer la temperatura corporal se eleva 5 décimas más de lo habitual debido al cambio del ciclo menstrual.

\section{Conclusión}

En conclusión, luego de comparar valores de temperaturas normales obtenidos en varios estudios, se ha concluido que la temperatura es un signo vital muy variable, y hay una gran diferencia entre los rangos normales de los pacientes pediátricos y adultos mayores. Se sabe que la temperatura está influenciada por varios factores tales como: la edad de la persona, el sitio de medición, el género, la hora del día, y también el tipo de dispositivo a usar. El estudio determina que el rango de temperatura normal oscila entre 36 y 37 grados centígrados, además se concluye que las mejores mediciones en la población involucrada son en la parte rectal.

Las medidas de la temperatura axilar, que se obtienen colocando un termómetro debajo de la axila, esta técnica no estaría re- comendada en los adultos porque se han mostrado imprecisas y reflejan mal la temperatura central. Sin embargo, las medidas axilares se han mostrado tan fiables como la medida de la temperatura rectal en los lactantes estables. Las temperaturas rectales suelen ser $0,5^{\circ} \mathrm{C}$ más altas que las orales y que los dispositivos usualmente utilizados son: los termómetros digitales.

Las zonas de temperatura oral, rectal y cutánea se apoyan en una circulación sanguínea eficaz. El calor de la sangre pasa a la sonda del termómetro. La temperatura timpánica se apoya en la radiación del calor corporal a un sensor infrarrojo. Como el tímpano comparte el mismo riego sanguíneo arterial que el hipotálamo, es una temperatura central; en cambio las medidas en la arteria temporal detectan la temperatura del flujo sanguíneo cutáneo.

\section{Recomendaciones}

Hay que destacar que la temperatura obtenida varía dependiendo del lugar usado. Cada lugar de medida de la temperatura tiene sus ventajas e inconvenientes por lo que aplicando el pensamiento crítico se deberá definir el lugar más seguro y preciso en cada paciente.

También cuando sea posible, se debe usar el mismo lugar para medidas repetidas si son necesarias, esto permitirá obtener medidas correctas intermitentes de la temperatura.

Por último, se hace necesario destacar que los resultados de los valores de la temperatura corporal pueden variar, debido a cambios en los métodos de medición introducidos por los dispositivos, técnicas incorrectas, entre otros, que puede no valorar con precisión el estado de salud o identificar alteraciones, por eso se debe realizar la toma de temperatura las veces que sean necesarias. 


\section{Bibliografía}

1. López M. Teoría y práctica de los fundamentos de enfermería (I). Bases teóricas y metodológicas [Internet]. Edual. 2020 [citado 22 Mayo 2021]. (12).147-148. Disponible en: https://www.researchgate.net/profile/CayetanoFernandezSola/publication/345894569_Teoria_y_practica_de_los_fundamentos_de_enfermeria_I_Bases_teoricas_y_metodologicas/links/5fb0e1d045851518fda6de1b/ Teoria-y-practica-de-los-fundamentos-deenfermeria-I-Bases-teoricas-y-metodologicas.pdf\#page $=46$

2. Duran A. Signos vitales. Mar del Plata [Internet]. Universidad Nacional de Mar del Plata. 2017 [citado 22 de Mayo 2021]. 2-5pag. Disponible en: file:///C:/users/client/downloads/modulo-signos-vitales-2017\%20(6).pdf.

3. Cervantes J, Reyes J, Bracho G. Desarrollo de un prototipo para su uso como herramienta de apoyo al personal médico en la gradación de triaje para pacientes en los servicios de urgencias basado en sus signos vitales [Internet]. Revista investigaciones andinas. 2017 [citado 29 Mayo 2021]. 34 (19); 1834. Disponible en: https://revia.areandina.edu. co/index.php/IA/article/view/936/783

4. Bruno S, Marracino, M, Tarducci G. Fisiología humana [Internet]. Universidad Nacional de la Plata. 2019 [citado 29 mayo 2021]. 4pag. Disponible en: https://memoria.fahce.unlp.edu.ar/programas/ pp.11404/pp.11404.pdf

5. Picquart M, Carrasco I. De la temperatura y su medición. Lat-Am J. [Internet]. Universidad Autónoma Metropolitana Unidad Iztapalapa. . 2017 [citado 3 Junio 2021]. 11(1):9-10.Disponible en: file:///C:/Users/client/Downloads/Dialnet-DeLaTemperaturaYSuMedicion-6019786\%20(3).pdf

6. Cajiga A, Aguirre C, Álvarez G, Escobedo L, Martínez A Labra A, García CG, Fernández J. Hipotermia postquirúrgica: incidencia en el Hospital Ángeles Lomas. [Internet]. Revista Médica Grupo Ángeles. 2020 [citado 3 Junio 2021]. 18(1); 112. Disponible en: https://www.medigraphic.com/ pdfs/actmed/am-2020/am201aa.pdf

7. Bohorquez A, Sánchez C, Carvajal S, Vargas N. Desarrollo e implementación con de un sistema de calibración con de termómetros clínicos infrarrojos de o ido. [Internet]. Revista de Física. 2020 [citado 9 Junio 2021]. 60 (7); 42-43 Disponible en: http:// www.scielo.org.co/pdf/momen/n60/0121-4470momen-60-41.pdf

8. Solís L, Jiménez A, Nieto $M$, Bolado C, Barrios M. Atención estomatológica: Selección de lecturas. Cirugía básica. La Habana-Cuba [Internet].
Ecimed ciencias médicas. 2020 [citado 12 Junio 2021]. 34(3); 159-161. Disponible en: http://aulavirtual.sld.cu/pluginfile.php/106586/mod_resource/content/1/atencion_estomatologia_completo. pdf\#page $=164$

9. Hermosín A, Pereira E, Loro D. Hipertermia en la tercera edad. [Internet]. Revista Electrónica de Portales Médicos Barcelona. 2017 [citado 12 Junio 2021]. Disponible en: https://www.revista-portalesmedicos.com/revista-medica/hipertermia-tercera-edad/

10. Picón Y, Orozco J, Molina J, Franky M. Control central de la temperatura corporal y sus alteraciones: fiebre, hipertermia e hipotermia. [Internet]. MedUNAB. 2020 [citado 9 Junio 2021]. 23(1); 125-127.Disponible en: https://docs.bvsalud.org/ biblioref/2020/04/1087985/3714.pdf

11. Giraudo F, Aros S, Handal G, Barrera F. Hipotermia y termorregulación en el niño. [Internet]. Revista boletín Chile. 2018 [citado 12 Junio 2021]. 17(72), 5-7. Disponible en: https://www.savalnet. cl/revistas/boletin_hcsba_abril_2018/files/assets/ common/downloads/Bolet.pdf

12. Rodrigues A, Coeli B, Pinheiro V, Rodrigues A, Vasconcelos E, Rodrigues A. Perfil de los recién nacidos de riesgo relacionados con la termorregulación en una Unidad de Cuidados Intensivos Neonatales. [Internet]. Revista Enfermería Global. 2021 [citado 12 Junio 2021]. 61 (8); 6064. Disponible en: https://scielo.isciii.es/pdf/eg/ v20n61/1695-6141-eg-20-61-59.pdf

13. Espina M. Perfil de los recién nacidos de riesgo relacionados con la termorregulación en una Unidad de Cuidados Intensivos Neonatales. [Internet]. Pontificia Universidad Católica del Ecuador. 2020 [citado 12 Junio 2021]. pag13-14. Disponible en: http://repositorio.puce.edu.ec/ handle/22000/17946

14. Romero J, Carrillo R, Meza J, Sosa J. Actualidades en el tratamiento de la fiebre en el paciente con sepsis y choque séptico: controversias y recomendaciones basadas en evidencia. [Internet]. Revista Medicina Interna México. 2017 [citado 18 agosto 2021]. 33(1); 102-103Disponible en: http:// www.scielo.org.mx/pdf/mim/v33n1/0186-4866mim-33-01-00099.pdf

15. Soengas N. Alteraciones en el control de la temperatura corporal en los adultos mayores. [Internet]. Revista Geriatría Clínica. . 2018 [citado 12 Junio 2021]. 12(2); 35-36.Disponible en: http://adm.meducatium.com.ar/contenido/articulos/16000340036_1125/pdf/16000340036.pdf 
16. Eggenberger $P$, Bürgisser $M$, Rossi R, Annaheim S. Body Temperature Is Associated With Cognitive Performance in Older Adults With and Without Mild Cognitive Impairment: A Cross-sectional Analysis. [Internet]. US National Library of Medicine National Institutes of Health. . 2021 [citado 18 Junio 2021]. 13pag Disponible en: https://www.ncbi.nlm. nih.gov/pmc/articles/PMC7907648/

17. Baca P, Bonilla K. Prototipo de un sistema de medición de temperatura corporal y pulso cardiaco, para registro médico digitalizado, en la clínica universitaria de becados internos de la UNAN-Managua [Internet]. Universidad Nacional Autónoma de Nicaragua, Managua. 2017 [citado 18 Junio 2021]. Disponible en: https://www.ncbi.nlm.nih. gov/pmc/articles/PMC7907648/

18. Sánchez J, Ramírez A, Tonguino A, Vargas L. Conocimiento del proceso de atención de enfermería por parte de los enfermeros de la Clínica La Inmaculada [Internet]. Revista de Sanidad Militar.2019 [citado 18 Junio 2021]. . 73(5); 278-28. Disponible en: file:///C:/Users/client/Downloads/ sm195_6b\%20(1).pdf

19. Alba F, Chinchay W. Plan de mantenimiento preventivo para mejorar la disponibilidad de equipos biomédicos - unidad cuidados intensivos, Hospital Víctor Ramos Guardia, Huaraz, 2018. Repositorio Institucional - UCV. [Internet]. Repositorio Institucional - UCV. 2019 [citado 18 Junio 2021]. Disponible en: file:///C:/Users/client/Downloads/ Alba_RFY-Chinchay_GWE.pdf

20. Alarcón A, Arias G, Miguel J. Sistema de medición de temperatura corporal a través de dispositivos móviles para monitorear niveles de fiebre en niños entre los 0 y 5 años en la ciudad de Neiva. [Internet]. Revista Internacional en Inteligencia Ambiental, Ingeniería de Software y Salud Electrónica y Móvil. 2018 [citado 18 Junio 2021]. 21(7); 21-22. Disponible en: https://revistas.utp.ac.pa/index.php/memoutp/article/view/1838/2685

21. Villanueva E, Fernández M, Viano E, Amorín M. Fiabilidad en la medición de la temperatura corporal con un termómetro timpánico en pacientes geriátricos. [Internet]. Gerokomos. 2017 [citado 18 Junio 2021]. 28 (2); 68-70. Disponible en: https:// scielo.isciii.es/pdf/geroko/v28n2/1134-928X-geroko-28-02-68.pdf

22. Storch P. Hipotermia. Golpe de calor. [Internet]. Sociedad Española de Urgencias de Pediatría (SEUP). . 2019 [citado 18 Junio 2021]. (3); 2-3.Disponible en: https://seup.org/pdf_public/pub/protocolos/23_Hipotermia.pdf

23. Reyes M, Casanova M, Aguirre D. Cuidados del bebe recién nacido sano. [Internet]. Revista científica del mundo de la investigación. . 2019 [citado 18 Junio 2021]. 4 (1); 396. Disponible en: https://www.recimundo.com/index.php/es/article/ view/766/1167

24. Quispe H. Efectividad del uso del termómetro digital versus el termómetro infrarrojo en el paciente pediátrico. [Internet]. Universidad Privada Norbert Wiener. 2020 [citado 18 Junio 2021]. (pag.1215)Disponible en: http://repositorio.uwiener.edu. pe/xmlui/bitstream/handle/123456789/3692/ T061_44342979_S.pdf?sequence=1\&isAllowe$\mathrm{d}=\mathrm{y}$.

25. Arias J. Constantes Vitales 2020 [Internet]. Universidad Tecnológica Fidel Fernández. . 2020 [citado 18 Junio 2021]. (pag.6-14). Disponible en: http:// ri.utn.edu.mx/bitstream/handle/123456789/601/ Constantes\%20Vitales\%202020.pdf?sequence=1

26. Marzuillo R, Webb D, Mohan J. Fever in Children: Pearls and Pitfalls. [Internet]. US National Library of Medicine National Institutes of Health. 2017 [citado 23 Junio 2021]. Disponible en: https://www. ncbi.nlm.nih.gov/pmc/articles/PMC8063696/

27. Thiel A, Evans A, Fuchs B, Arnemo, J. Effects of reproduction and environmental factors on body temperature and activity patterns of wolverines [Internet]. US National Library of Medicine National Institutes of Health. 2019 [citado 23 Junio 2021]. 16 (21) Disponible en: https://www.ncbi.nlm.nih. gov/pmc/articles/PMC6580505

28. Dan L, Kasper D, Jameson, L, Fauci A. Alteraciones de la temperatura corporal | Harrison. Principios de Medicina Interna. [Internet]. McGraw-Hill Medical. 2017 [citado 23 Junio 2021]. Disponible en: https://accessmedicina.mhmedical.com/content. aspx?bookid $=1622$ \&sectionid $=101813532$

29. Fernández A, Herrera J, Sibaja J. Perfil nacional de uso de mercurio en Costa Rica a la luz de la entrada en vigencia del Convenio de Minamata. [Internet]. Revista de Ciencias Ambientales. 2017 [citado 23 Junio 2021]. Disponible en: https://www. revistas.una.ac.cr/index.php/ambientales/article/ view/9484/11251

30. Alatrista A, Romero R, Baldeón K, Arias C. Knowledge and practices on reduction of mercury added to medical devices in health workers [Internet]. Revista de la Facultad de Medicina Humana. 2018 [citado 27 Junio 2021]. 8(4); Disponible en: http://revistas.urp.edu.pe/index.php/RFMH/article/ view/1729/1643

31. Zarate, J. Fiebre. Revista de divulgación científica de nutrición ambiental y seguridad alimentaria. (2017) [citado 27 Junio 2021]. 6 (2); 17.Disponible en: https://www.ugto.mx/redicinaysa/images/ 
publicaciones/2017/Vol.-6-No.-2.pdf\#page=15

32. Quebrado C., Dolores, T. (2019). Incidencia de hipotermia intraoperatoria en pacientes geriátricos sometidos a cirugía abdominal con y sin medidas antihipotermia en el Hospital de especialidades de Puebla. benemérita Universidad Autónoma de Puebla. (2019). [citado 27 Junio 2021] (Pag.1019).

33. Gonzales, J, Carrillo, R, Meza, J, García, J. (2017). Actualidades en el tratamiento de la fie- bre en el paciente con sepsis y choque séptico: controversias y recomendaciones basadas en evidencia. Revista Medicina Interna México; (2017). [citado 27 Junio 2021]. 33(1); 102-103.Disponible en:http://www.scielo.org.mx/pdf/mim/v33n1/01864866-mim-33-01-00099.pdf

34. Rivadeneyra E, Molina T, Bernal. Variaciones cíclicas de la temperatura corporal en la mujer. [Internet]. guía de prácticas de morfofisiología. 2020 [citado 27 Junio 2021]. 56-61.Disponible en: https://www.uv.mx/qfb/files/2020/09/Guia-de-Morfofisiologia.pdf\#page $=63$

\section{CITAR ESTE ARTICULO:}

Mena Sánchez, S. M., \& Quenorán Almeida, V. S. (2021). Valores de la temperatura en pacientes pediátricos y adultos mayores. "un enfoque de revisión”. RECIMUNDO, 5(4), 332-343. https://doi.org/10.26820/recimundo/5.(4). dic.2021.332-343

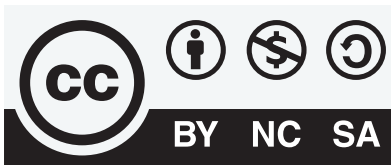

CREATIVE COMMONS RECONOCIMIENTO-NOCOMERCIAL-COMPARTIRIGUAL 4.0 . 